\title{
The use of ultra-widefield retinal imaging in the setting of de Morsier Syndrome
}

\author{
Ursula M Anders ${ }^{1,2}$ and James B Aguayo-Martel ${ }^{1-4 *}$ \\ ${ }^{1}$ Martel Eye Medical Group, Rancho Cordova, CA, USA \\ ${ }^{2}$ Leading Medical Technologies, Inc., Rancho Cordova, CA, USA \\ ${ }^{3}$ California Northstate University College of Medicine, Elk Grove, CA, USA \\ ${ }^{4}$ Dignity Health, Carmichael, CA, USA
}

\begin{abstract}
We describe the utilization of ultra-widefield retinal imaging in a patient suffering from de Morsier syndrome, with known pituitary and optic nerve hypoplasia with secondary congenital nystagmus, who developed late onset diabetes type II. Due to the nystagmus it was not possible to assess the patient and monitor for the development of diabetic retinopathy by standard retinal imaging and tomography. Aspects of the de Morsier syndrome with relationship to secondary obesity and subsequent diabetes type II will be discussed, as well as the utility of opto-mapping in this setting.
\end{abstract}

\section{Introduction}

de Morsier syndrome, now known as septo-optic dysplasia, was initially outlined by David Reeves, and later by Georges de Morsier who described the condition as being associated with the corpus callosum, septum pellucidum, pituitary gland dysfunction, and optic nerve hypoplasia $[1,2]$. Current definitional standards are that only two of the criteria need to be met to confirm the diagnosis of this syndrome. Disruption to transcriptional factors HESX1, SOX2, SOX3, and OTX2 are thought to contribute to the abnormal midline and pituitary development in septo-optic dysplasia $[3,4]$. The condition is known to affect pituitary function in $62 \%$ of cases that are tested [5]. This condition can lead to a number of abnormalities, which require lifelong monitoring by endocrinologists. The severity of optic nerve hypoplasia secondarily leads to a congenital nystagmus, which must be continually monitored by a neuroophthalmologist. Optic nerve hypoplasia is commonly associated with hypothalamic dysfunction, which can lead to problems of obesity. Hyperphagia with obesity may affect this population. This may be due to dysfunction of the hypothalamic ventromedial nuclei, which stimulate hunger in response to leptin, and the lateral nuclei, which stimulate feeding behavior and regulate metabolism [6]. The association of obesity to type II diabetes is well-known.

Due to the nystagmus, it has been difficult to obtain fundoscopic or optical coherent tomographic imaging, which are necessary for patients with this condition. The use of ultra-widefield (UWF) retinal imaging utilizing scanning laser ophthalmoscopy as a means to obtain very high fidelity and detailed images are deemed to be vital for patients with diabetic retinopathy $[7,8]$. UWF has the ability to monitor background diabetic retinopathy changes such as hemorrhaging, cotton wool spots, intraretinal hemorrhaging, or possible retinal neovascularization. It can also be of great utility in assessing the optic nerve and its changes over time. We describe a case where this instrument was capable of aiding in the diagnosis of diabetic retinopathy in a patient who was previously felt to be retinopathy free.

\section{Case}

A 37 year old male, with a history of diabetes mellitus type 2, obesity, hypertension, hyperlipidemia, panhypopituitarism, and septooptic dysplasia. He had been followed for a 16 year duration at yearly intervals. A CT of the brain revealed a small sella turcica, and a small infundibulum with hypoplasia of the pituitary gland. An MRI of the brain was also obtained, revealing decreased pituitary parenchyma within a small sella turcica. A soft tissue nodule in the hypothalamic region measuring approximately $8 \mathrm{~mm}$ in diameter, was presumed to represent a regenerated or ectopic posterior pituitary. The corpus callosum and septum pellucidum were normal in appearance, however the optic chiasm was hypoplastic (Figure 1). The patient was also followed by neuro-endocrinology and neuro-ophthalmology at the University of California, San Francisco and endocrinology at the University of California, Davis. The patient was presumed to have no diabetic retinopathy by ophthalmology or neuro-ophthalmology, since the diagnosis. Previous attempts of fundus photography and optical coherence tomography were unsuccessful.

Current ophthalmologic examination revealed uncorrected vision of hand motion in the right eye and count fingers in the left eye, not improved with correction. Intraocular pressures were $17 \mathrm{mmHg}$ in the right eye and $18 \mathrm{mmHg}$ in the left. Extraocular movement revealed congenital nystagmus in both eyes. Other than mild nuclear sclerotic cataracts, external, and anterior segment examination was

Correspondence to: James Benjamin Aguayo-Martel, Clinical Professor of Ophthalmology, California Northstate University College of Medicine, $9700 \mathrm{~W}$ Taron Drive, Elk Grove, CA 95757, USA; Tel: (916) 686-7300; Fax: (916) 6867310; E-mail: james.martel@cnsu.edu

Key words: de Morsier, septo-optic dysplasia, pituitary hypoplasia, optic nerve hypoplasia, nystagmus, optomap, diabetes mellitus

Received: May 18, 2016; Accepted: June 02, 2016; Published: June 06, 2016 


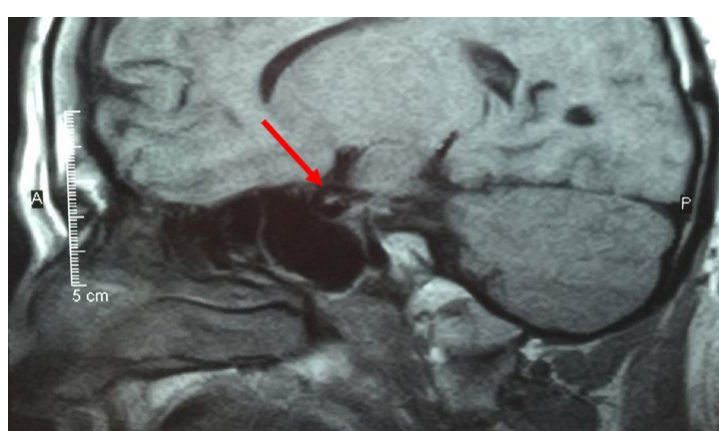

Figure 1: Sagittal view of an MRI of the brain.

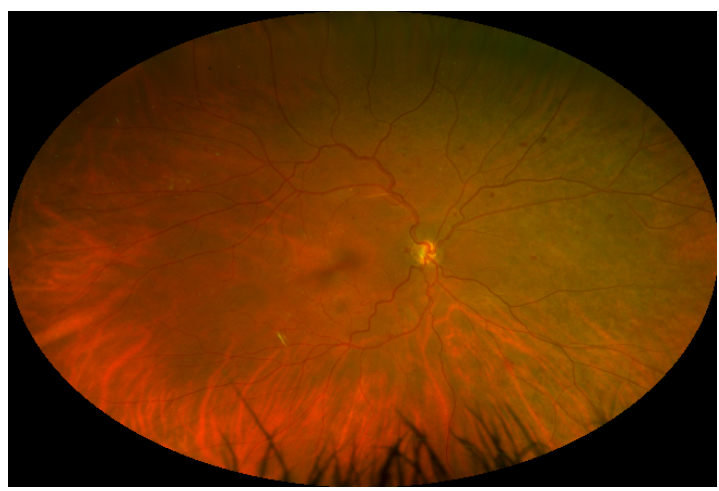

Figure 2: Ultra-widefield retinal imaging of the right eye.

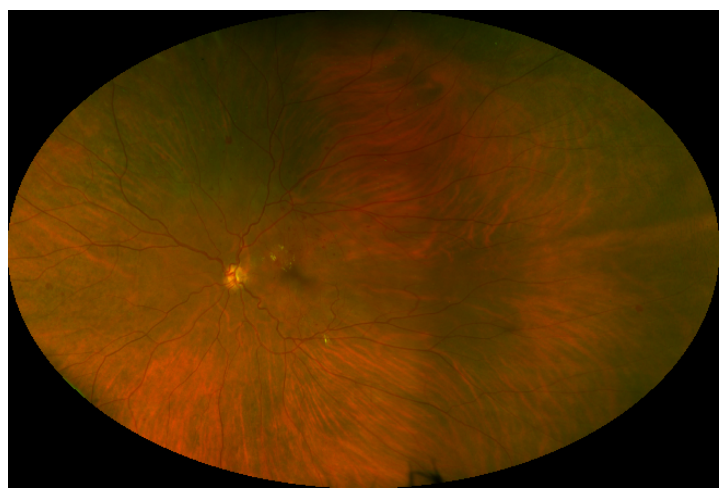

Figure 3: Ultra-widefield retinal imaging of the left eye. unremarkable. Fundus photography was obtained by a California ultra-widefield imaging system (Optos, Dunfermline, Scotland, UK). This revealed bilateral optic nerve hypoplasia with pale optic nerves, venous tortuosity, temporal straightening of the retinal vasculature, retinal exudates, retinal microaneurysms, and intraretinal hemorrhages indicative of diabetic retinopathy (Figures 2 and 3).

\section{Discussion}

The nature of de Morsier syndrome can have devastating consequences on patients, from both an endocrinological as well as a visual perspective. A new technique has been developed for monitoring diabetic retinopathy and other retinal peripheral diseases using a scanning laser ophthalmoscope. With this technique a small focal point is created behind the lens, which then allows for a complete mapping of the retina within one to two seconds (producing an optomap). In this article we discuss the first such presentation of a case of de Morsier syndrome with secondary diabetes type II, which demonstrates the extreme usefulness of this imaging modality. We feel it will have great utility, certainly in this patient population as well as in the diabetic population as a whole.

\section{References}

1. Reeves DL (1941) Congenital absence of the septum pellucidum. Bull Johns Hopkins Hosp 69: 61-71.

2. deMorsier G (1956) Studies on malformation of cranio-encephalic sutures. III Agenesis of the septum lucidum with malformation of the optic tract. Schweiz Arch Neurol Psychiatr 77: 267-292.[Crossref]

3. McCabe MJ, Alatzoglou KS, Dattani MT (2011) Septo-optic dysplasia and other midline defects: the role of transcription factors: HESX1 and beyond. Best Pract Res Clin Endocrinol Metab 25: 115-124.[Crossref]

4. Polizzi A, Pavone P, Iannetti P, Manfre L, Ruggieri M (2006) Septo-optic dysplasia complex: a heterogenous malformation syndrome. Pediatr Neurol 34: 66-71.[Crossref]

5. Morishima A, Aranoff GS (1986) Syndrome of septo-optic-pituitary dysplasia: The clinical spectrum. Brain Dev 8: 233-239.[Crossref]

6. Garcia-Filion P, Borchert M (2013) Optic nerve hypoplasia syndrome: A review of the epidemiology and clinical associations. Curr Treat Options Neurol 15:78-89.[Crossref]

7. Silva PS, Cavallerano JD, Haddad NM, Kwak H, Dyer KH, et al. (2015) Peripheral lesions identified on ultrawide field imaging predict increased risk of diabetic retinopathy progression over 4 years. Ophthalmology 122: 949-956.[Crossref]

8. Silva PS, Cavallerano JD, Sun JK, Noble J, Aiello LM, et al (2012) Nonmydriaticultrawide field retinal imaging compared with dilated standard 7-field $33-\mathrm{mm}$ photography and retinal specialist examination for evaluation of diabetic retinopathy. Am J Ophthalmol 154: 549-59.e2.[Crossref]

Copyright: (2016 Anders UM. This is an open-access article distributed under the terms of the Creative Commons Attribution License, which permits unrestricted use, distribution, and reproduction in any medium, provided the original author and source are credited. 